\title{
Nonexistence of a Shock Layer in Gas Dynamics with a Nonconvex Equation of State
}

\author{
Robert L. PeGo \\ Communicated by J. SERRIN
}

\begin{abstract}
A classical result of GILBARG states that a simple shock wave solution of Euler's equations is compressive if and only if a corresponding shock layer solution of the Navier-Stokes equations exists, assuming, among other things, that the equation of state is convex. An "entropy condition" appropriate for weeding out "unphysical" shocks in the nonconvex case has been introduced by T.-P. LIU. For shocks satisfying his entropy condition, LIU showed that purely viscous shock layers exist (with zero heat conduction). Dropping the convexity assumption, but retaining many other reasonable restrictions on the equation of state, we construct an example of a (large amplitude) shock which satisfies Liu's entropy condition but for which a shock layer does not exist if heat conduction dominates viscosity. We also give a simple restriction, weaker than convexity, which does guarantee that shocks which satisfy. Liu's entropy condition always admit shock layers.
\end{abstract}

\section{Introduction}

In 1951 GILBARG [2] showed that for each stationary shock wave solution of Euler's equations of gas dynamics in one space dimension there exists a corresponding smooth solution (called a shock layer or shock profile) of the NavierStokes equations with viscosity and heat conduction, provided that the thermodynamic equation of state satisfies a short list of restrictions given by WEYL [11], including a convexity condition. The shock wave is required to satisfy the Rankine-Hugoniot jump relations and the entropy condition, which ensures that entropy increases along particle paths. Under WEYL's restrictions, the entropy condition simply requires shocks to be compressive, i.e., density must increase along particle paths.

More recently, there has been considerable interest in equations of state which may be nonconvex ([5], [9], [10]). T.-P. LiU introduced an entropy condition appropriate for shocks in this situation. He showed that a shock satisfies 
his entropy condition if and only if a purely viscous shock layer exists, i.e., a shock layer for the equations with zero heat conduction [4].

The purpose of this note is to present an example concerning restrictions on the equation of state under which Liu's entropy condition does select just those shocks for which shock layers exist for any positive values of viscosity and heat conduction. We explicitly construct an equation of state such that for some shock wave which satisfies Liu's entropy condition, no corresponding shock layer can exist if the heat conduction dominates the viscosity. (A shock layer does exist if heat conduction is small compared to viscosity.) The equation of state satisfies all of WeYL's restrictions except the convexity condition, and satisfies other conditions which have appeared in recent work on the Riemann problem in gas dynamics ([5], [10]). We can give a simple restriction weaker than convexity (satisfied by van der Waals gases in regions of hyperbolicity, for example) under which all shocks satisfying LiU's entropy condition always admit corresponding shock layers, see Theorem 1.

Examples of the type described here may be of interest in one dimensional thermo(visco)elasticity, where for quite general constituitive relations it is desirable to determine the smoothing effect of viscous and thermal dissipation (e.g. see [1]). Nonconvex equations of state also occur for materials exhibiting phase transitions. In this paper, however, we require that Euler's equations remain hyperbolic in the region of interest. Effects other than viscosity and heat conduction must be considered to determine "structure" in phase transition zones, see [8] and [9].

The Navier-Stokes equations in one space dimension, written in Lagrangian form, are

$$
\begin{gathered}
\tau_{t}-u_{h}=0 \\
u_{t}+p_{h}=\left(\mu u_{h} / \tau\right)_{h} \\
\mathscr{E}_{t}+(p u)_{h}=\left(\mu u u_{h} / \tau\right)_{h}+\left(\lambda \theta_{h} / \tau\right)_{h} .
\end{gathered}
$$

Here $h$ denotes the Lagrangian mass coordinate, $t$ is time, $\tau$ is specific volume, $u$ is velocity, $p$ is pressure, $\theta$ is temperature, $\mathscr{E}$ is energy density per unit mass, $\mu$ and $\lambda$ are the coefficients of viscosity and heat conduction, respectively, and $\mathscr{E}=e+u^{2} / 2$, where $e$ is the internal energy density per unit mass. We assume that $\tau$ and $\theta$ determine the thermodynamic state of the material, and that $e, p$, and the entropy $S$ are given by sufficiently smooth equations of state $e=e(\tau, \theta)$, $p=p(\tau, \theta), S=S(\tau, \theta)$. These functions are related through the Gibbs relation

$$
\theta d S=d e+p d \tau
$$

The coefficients $\mu$ and $\lambda$ may also depend smoothly on $\tau$ and $\theta$. The quantities $\mu, \lambda, \tau, \theta$, and $p$ are positive. For convenience, we shall denote the triple $(\tau, u, \mathscr{E})$ by $U$, and the thermodynamic state by $Z$, specified either by the pair $(\tau, \theta)$ or by $(\tau, p)$, see assumption (1.10) below.

Euler's equations are obtained from (1.1) by setting $\mu=\lambda=0$. A shock wave traveling at speed $s$ is a weak solution of these equations of the form

$$
U(h, t)=\left\{\begin{array}{ll}
U_{-} & \text {for } h<s t \\
U_{+} & \text {for } h>s t
\end{array} .\right.
$$


The two end states must satisfy the Rankine-Hugoniot relations

$$
\begin{gathered}
0=-s\left(\tau_{+}-\tau_{-}\right)-\left(u_{+}-u_{-}\right) \\
0=-s\left(u_{+}-u_{-}\right)+\left(p_{+}-p_{-}\right) \\
0=-s\left(\mathscr{E}_{+}-\mathscr{E}_{-}\right)+\left(p_{+} u_{+}-p_{-} u_{-}\right) .
\end{gathered}
$$

We call any solution (1.3) satisfying (1.4) a "simple jump solution", or a "jump". It is customary to reserve the term "shock" for jumps which satisfy the entropy condition.

What determines a jump? The speed $s$ may be determined up to sign from any suitable pair of thermodynamic states $Z_{+}$and $Z_{-}$. by the relation

$$
0=s^{2}\left(\tau_{+}-\tau_{-}\right)+\left(p_{+}-p_{-}\right) .
$$

In our analysis it suffices to consider only back-facing shocks, for which $s<0$. (Particle paths in (1.1) are vertical.) Now $u_{+}-u_{-}$is determined. From the third equation of (1.4) we next find that the states $Z_{+}$and $Z_{-}$determine a simple jump solution with $s<0$ if and only if the Hugoniot function vanishes, that is

$$
H\left(Z_{+}, Z_{-}\right) \equiv e_{+}-e_{-}+\frac{1}{2}\left(p_{+}+p_{-}\right)\left(\tau_{+}-\tau_{-}\right)=0 .
$$

with $Z_{0}$ fixed, the set of states $Z$ satisfying $H\left(Z, Z_{0}\right)=0$ is typically a curve, called the Hugoniot curve with center $Z_{0}$ in the $(\tau, p)$ plane.

A shock layer for the jump (1.3) is a traveling wave solution $U=U(h-s t)$ of (1.1) with $U(\xi) \rightarrow U_{ \pm}$as $\xi \rightarrow \pm \infty$. With this assumption, a further integration shows that $U(\xi)$ must satisfy

$$
\begin{gathered}
0=-s\left(\tau-\tau_{ \pm}\right)-\left(u-u_{ \pm}\right) \\
\mu u_{\xi} / \tau=-s\left(u-u_{ \pm}\right)+\left(p-p_{ \pm}\right) \\
\mu u u_{\xi} / \tau+\lambda \theta_{\xi} / \tau=-s\left(\mathscr{E}-\mathscr{E}_{ \pm}\right)+p u-p_{ \pm} u_{ \pm} .
\end{gathered}
$$

The first equation determines $u(\xi)$ from $\tau(\xi)$, so we obtain

$$
\begin{aligned}
& \lambda \theta_{\xi} / \tau=-s\left(\mathscr{E}-\mathscr{E}_{ \pm}+\left(\tau-\tau_{ \pm}\right)\left(p_{ \pm}+s u\right)\right) \equiv L(\tau, \theta) \\
& \mu \tau_{\xi} / \tau=-\frac{1}{s}\left[s^{2}\left(\tau-\tau_{ \pm}\right)+p-p_{ \pm}\right] \equiv M(\tau, \theta) .
\end{aligned}
$$

These equations yield an autonomous system of ordinary differential equations in the $(\tau, \theta)$ plane. It is clear that $Z_{+}$and $Z_{-}$are rest points for this system. A shock layer exists for the jump (1.2) if and only if the system (1.5) admits a trajectory $Z(\xi)$ connecting $Z_{-}$to $Z_{+}$, i.e., $Z(\xi) \rightarrow Z_{ \pm}$as $\xi \rightarrow \pm \infty$.

We remark that equations (1.6) are identical to those used by GILBARG [2] for the stationary shock layer in Eulerian form, if we introduce an Eulerian space variable $x=\int^{\xi} \tau(\eta) d \eta$ and recognize that $-s$ is the mass flux $\varrho u=$ const, where $\varrho=1 / \tau$ is the density.

In order to motivate LiU's entropy condition for the jump (1.3) and examine the implications of nonconvexity, we briefly consider the simple example of 
isothermal gas dynamics in Lagrangian form. The equations are

$$
\begin{gathered}
\tau_{t}-u_{h}=0 \\
u_{t}+p(\tau)_{h}=\left(\mu u_{h} / \tau\right)_{h} .
\end{gathered}
$$

A simple jump with speed $s$ connecting $\left(\tau_{-}, u_{-}\right)$and $\left(\tau_{+}, u_{+}\right)$for $\mu=0$ satisfies

$$
0=s^{2}\left(\tau_{+}-\tau_{-}\right)+p\left(\tau_{+}\right)-p\left(\tau_{-}\right) .
$$

A shock profile $(\tau, u)(\xi)$, with $\xi=h-s t$, must satisfy (eliminating $u$ )

$$
\mu \tau_{\xi} / \tau=-\frac{1}{s}\left[s^{2}\left(\tau-\tau_{ \pm}\right)+p(\tau)-p\left(\tau_{ \pm}\right)\right] \equiv M(\tau) .
$$

Assuming that $p(\tau)$ is decreasing, but possibly nonconvex, any two states $\tau_{+}, \tau_{-}$ determine a jump with speed $s<0$. A shock layer exists for that jump if and only if $\operatorname{sgn} M(\tau)=\operatorname{sgn}\left(\tau_{+}-\tau_{-}\right)$for $\tau$ between $\tau_{+}$and $\tau_{-}$. This is the "entropy condition" which selects those jumps which admit a shock layer for these equations. The situation is illustrated in Figure 1, where the phase portrait of (1.7) is indicated on the $\tau$ axis of the graph of $p(\tau)$. Observe that since $p$ is not convex, rarefaction shock layers can possibly exist, e.g. $\tau_{-}=\tau_{2}<\tau_{+}=\tau_{3}$. Also, a compressive jump need not admit a shock layer, e.g. $\tau_{-}=\tau_{4}>\tau_{+}=\tau_{1}$.

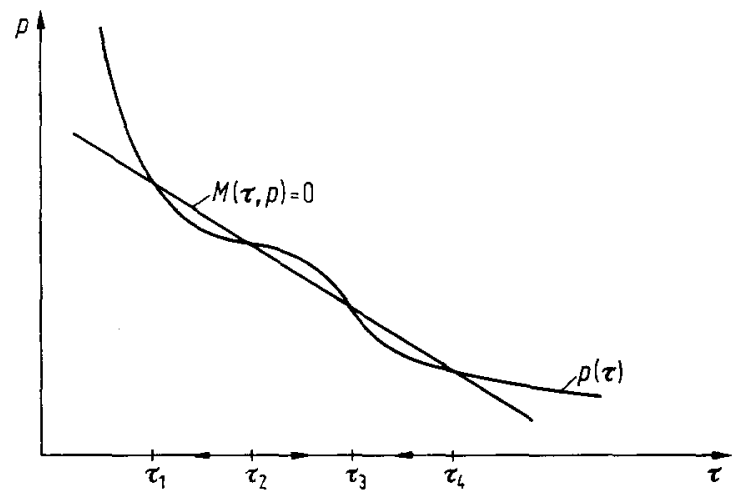

Fig. 1. Shock profiles in isothermal gas dynamics

For the non-isothermal case Liu's strict entropy condition for a jump (1.3) determined by $Z_{+}, Z_{-}$is that (with $s<0$ )

$\begin{array}{ll} & \operatorname{sgn} M(Z)=\operatorname{sgn}\left(\tau_{+}-\tau_{-}\right) \text {for all } Z=(\tau, p) \\ (E)_{s} & \text { between } Z_{+} \text {and } Z_{-} \text {on the Hugoniot curve }\end{array}$ with center $Z_{+}$(or with center $Z_{-}$).

Since $s<0$, this condition simply means that the line $M(\tau, p)=s^{2}\left(\tau-\tau_{ \pm}\right)+$ $p-p_{ \pm}=0$ should lie above (below) the Hugoniot curve between $Z_{+}$and $Z_{-}$ if $\tau_{-}>\tau_{+}\left(\tau_{-}<\tau_{+}\right)$. The restrictions we shall impose on the equation of state imply that, with some choice of center, the Hugoniot curve is regular between $Z_{+}$and $Z_{-}$, so the condition $(E)_{s}$ is well defined. 
The restrictions we place on the equation of state are as follows:

$$
e_{\theta}(\tau, \theta)>0
$$

this means that heat conduction is a dissipative effect in (1.1) for $\lambda>0$.

$$
p_{\tau}(\tau, S)<0
$$

Euler's equations are strictly hyperbolic just when this holds.

$$
p_{\theta}(\tau, \theta)>0
$$

thus the map $(\tau, \theta) \mapsto(\tau, p(\tau, \theta))$ is one-to-one on the domain of states $\Omega_{\theta}$. The image of this map we designate $\Omega_{p}$. We assume finally that

$$
\Omega_{p} \text { is convex. }
$$

These restrictions are the same as those imposed by GiLBARG [2], following WEYL [11], except that we have omitted the usual convexity assumption

$$
p_{\tau \tau}(\tau, S)>0 \text {. }
$$

Under these restrictions, the Hugoniot curve is described by the following proposition, whose proof is deferred until Section 2 .

Proposition 1. Fix $Z_{0}=\left(\tau_{0}, p_{0}\right)$ in $\Omega_{p}$. In the region $\tau \geqq \tau_{0}$ the set $\left\{Z \in \Omega_{p} \mid H\left(Z, Z_{0}\right)=0\right\}$ consists solely of a monotonically decreasing curve $p=h_{0}(\tau)$ passing through $Z_{0}$.

We now describe our positive results concerning existence and uniqueness of the shock layer for any given coefficients $\mu, \lambda$ which are positive functions of $\tau$ and $\theta$.

Theorem 1. Assume (1.8)-(1.11), and also

$$
e_{\tau}(\tau, \theta) \geqq 0 \quad \text { in } \Omega_{\theta} .
$$

Suppose $Z_{+}, Z_{-}$in $\Omega_{\theta}$ satisfy $H\left(Z_{+}, Z_{-}\right)=0$ and (1.13) below. Then the simple jump solution determined by $Z_{+}, Z_{-}$and $s<0$ from (1.5) admits a unique shock layer if and only if the strict entropy condition $(E)_{s}$ is satisfied.

In the absence of (1.12), compressive shocks (that is, $\tau_{-}>\tau_{+}$if $s<0$ ) always admit a shock layer, as shown by the following

Theorem 2. Assume (1.8)-(1.11). Suppose $Z_{+}, Z_{-}$in $\Omega_{\theta}$ satisfy $H\left(Z_{+}, Z_{-}\right)=0$ and (1.13) below, and suppose $\tau_{-}>\tau_{+}$. Then the conclusion of Theorem 1 holds.

Weak shocks $\left(\left|U_{+}-U_{-}\right|\right.$small) always admit a shock layer, under the most basic of restrictions. This is shown by

Theorem 3. Assume only (1.8), (1.9). Fix $Z_{0}$ in $\Omega_{\theta}$. Then if $Z_{+}$and $Z_{-}$are sufficiently close to $Z_{0}$ and satisfy $H\left(Z_{+}, Z_{-}\right)=0$, the simple jump solution 
determined by $Z_{+}, Z_{-}$with $s<0$ admits a shock layer (unique in a neighborhood of $Z_{0}$ ) if and only if the strict entropy condition $(E)_{s}$ is satisfied.

Theorem 3 is proved in [7] as a consequence of a general theorem on singular viscosity matrices for systems of conservation laws. The proof of Theorems 1 and 2 is similar to that of Gilbarg [2], and will be presented briefly in Section 2. The extra condition imposed is a technical "domain" condition,

The line segment $\left[\tau_{+}, \tau_{-}\right] \times\left\{\theta_{-}\right\}$lies in $\Omega_{\theta}$,

needed so that certain constructions are valid. (A similar assumption was tacitly made by GILBARG.)

Our main result is the following "couterexample"

Theorem 4. There exists an equation of state defined in a domain $\Omega_{\theta}$ and satisfying (1.8)-(1.11), and a pair of states $Z_{+}, Z_{-}$which satisfy $H\left(Z_{+}, Z_{-}\right)=0$ and (1.13), such that the jump solution determined by $Z_{+}, Z_{-}$and $s(<0)$ satisfies the strict entropy condition $(E)_{s}$.

Moreover if $\lambda / \mu$ is sufficiently large, no corresponding shock layer exists, while if $\lambda / \mu$ is sufficiently small, a unique shock layer exists.

From Theorem 2 this shock must be a rarefaction shock, namely $\tau_{-}<\tau_{+}$. We stress, however, that LiU's result shows that a purely viscous shock layer (with $\lambda=0$ ) exists, so in fact the entropy does increase across this shock, i.e., $S\left(Z_{-}\right)<S\left(Z_{+}\right)$.

The equation of state in Theorem 4 must violate (1.12) and the convexity condition. However, the following conditions can be satisfied:

$$
\begin{aligned}
e_{\tau}(\tau, p) & \geqq 0 \\
p_{\tau}(\tau, \theta) & \leqq 0 .
\end{aligned}
$$

Condition (1.14) (along with (1.8)-(1.10)) was imposed by LIU [5] to guarantee that the Riemann problem for gas dynamics has a unique solution globally. Condition (1.15), along with (1.8), implies (1.9) and furthermore shows that constant states for the Navier-Stokes equations are linearly stable, see [6] and [7].

Finally, we briefly remark that although we have presented this example in the context of gas dynamics, it is valid in the context of one-dimensional thermoviscoelasticity as well. One may redefine the equation of state outside a compact domain so that the restrictions imposed by DAFERMOs [1] are satisfied. The key step is to provide an appropriate global specification for the function $p_{\theta}(\tau, \theta)$, cf. section 3 .

\section{Existence and uniqueness of the shock layer}

We begin this section by gathering several useful thermodynamic calculations, following which we prove Theorems 1 and 2 . Take $\tau$ and $\theta$ as independent variables. Using (1.2), and equating mixed partial derivatives of $S(\tau, \theta)$, we obtain 
the standard identities

$$
\begin{gathered}
\theta S_{\theta}(\tau, \theta)=e_{\theta}(\tau, \theta) \\
\theta S_{\tau}(\tau, \theta)=e_{\tau}(\tau, \theta)+p=\theta p_{\theta}(\tau, \theta) .
\end{gathered}
$$

Considering $\tau$ and $p$ independent, we get

$$
\begin{gathered}
\theta S_{p}(\tau, p)=e_{p}(\tau, p)=e_{\theta}(\tau, \theta) \theta_{p}(\tau, p) \\
\theta S_{\tau}(\tau, p)=e_{\tau}(\tau, p)+p
\end{gathered}
$$

and also

$$
p_{\tau}(\tau, S)=-S_{\tau}(\tau, p) / S_{p}(\tau, p) .
$$

The assumptions (1.8)-(1.10) imply that the derivatives $S_{\theta}(\tau, \theta), S_{\tau}(\tau, \theta), S_{p}(\tau, p)$ and $S_{\tau}(\tau, p)$ are all positive.

The following identity relates the entropy condition $(E)_{s}$ to the ordinary differential equations (1.6).

Proposition 2. Fix $Z_{+}$and $Z_{-}$with $H\left(Z_{+}, Z_{-}\right)=0$. Let $s<0$ satisfy (1.5). Hence $L(\tau, \theta)$ and $M(\tau, \theta)$ in (1.6) are determined, and for any $Z=(\tau, \theta)$ we have

$$
H\left(Z, Z_{ \pm}\right)=-\frac{1}{s} L(Z)+\frac{1}{2}\left(\tau-\tau_{ \pm}\right)(-s M)(Z)
$$

Finally

$$
H\left(Z, Z_{+}\right)-H\left(Z, Z_{-}\right)=\frac{1}{2}\left(\tau_{-}-\tau_{+}\right)(-s M)(Z) .
$$

Proof. Recalling that $u-u_{ \pm}=-s\left(\tau-\tau_{ \pm}\right)$, we have

$$
\begin{gathered}
H\left(Z, Z_{ \pm}\right)+L(Z) / s=-\frac{1}{2}\left(u^{2}-u_{ \pm}^{2}\right)+\left(\tau-\tau_{ \pm}\right)\left[\frac{1}{2}\left(p+p_{ \pm}\right)-p_{ \pm}+s u\right] \\
=\left(\tau-\tau_{ \pm}\right)\left[\frac{1}{2} s\left(u+u_{ \pm}\right)+\frac{1}{2}\left(p-p_{ \pm}\right)-s u\right]=\frac{1}{2}\left(\tau-\tau_{ \pm}\right)(-s M)(Z) .
\end{gathered}
$$

Proof of Proposition 1. With $Z_{0}$ fixed, $H\left(Z, Z_{0}\right)$ is a function of $Z=(\tau, p)$. From (2.2) it follows that

and

$$
H_{\tau}(\tau, p)=\theta S_{r}(\tau, p)+\frac{1}{2}\left(p_{0}-p\right)>0 \quad \text { if } p<p_{0},
$$

$$
H_{p}(\tau, p)=e_{p}(\tau, p)+\frac{1}{2}\left(\tau-\tau_{0}\right)>0 \quad \text { if } \tau \geqq \tau_{0} .
$$

It is convenient next to observe that

$$
\begin{gathered}
-\frac{1}{s} L_{\theta}(\tau, \theta)=e_{\theta}(\tau, \theta) \quad-\frac{1}{s} L_{\tau}(\tau, \theta)=\theta p_{\theta}(\tau, \theta)+s M(\tau, \theta) \\
-s M_{\theta}(\tau, \theta)=p_{\theta}(\tau, \theta) \quad-s M_{\tau}(\tau, \theta)=s^{2}+p_{\tau}(\tau, \theta) .
\end{gathered}
$$

To get $L_{\tau}$, use Proposition 2 and (2.2). 
Proof of Theorem 2. Let $Z_{+}, Z_{-}$in $\Omega_{\theta}$ satisfy $H\left(Z_{+}, Z_{-}\right)=0$ together with the condition (1.13) and $\tau_{-}>\tau_{+}$, and let $s<0$ satisfy (1.5). Suppose first that the entropy condition $(E)_{s}$ is satisfied. Our proof, like GILBARG's, is based on an analysis of the curves $L=0$ and $M=0$ which pass through $Z_{+}$and $Z_{-}$. Since $L_{\theta}$ and $M_{\theta}$ are positive, $\theta$ is a given function of $\tau$ along each of these curves, $\theta=l(\tau), \theta=m(\tau)$ respectively. We claim that $l(\tau)$ and $m(\tau)$ are defined for $\tau_{+} \leqq \tau \leqq \tau_{-}$and satisfy

$$
\begin{gathered}
l(\tau)<m(\tau) \quad \text { for } \tau_{+}<\tau<\tau_{-} \\
l^{\prime}(\tau)<0 \quad \text { for } \tau_{+} \leqq \tau \leqq \tau_{-} .
\end{gathered}
$$

Indeed, the set $\{M=0\}$ in $\Omega_{p}$ is a straight line segment, by (1.11). Pulling back to the $(\tau, \theta)$ plane, $m(\tau)$ is defined as described. Using Proposition 2, the entropy condition $(E)_{s}$ implies that $L(Z)>0>M(Z)$ between $Z_{+}$and $Z_{-}$on the Hugoniot curve with center $Z_{+}$. Property (i) follows at once. Property (ii) is obtained from (i) by using (2.7), since $M \leqq 0$ on the curve $L=0$. The "domain" condition (1.13), with (1.11), now implies that $l(\tau)$ is defined as described.

The existence of the shock layer follows from (i), (ii), see Figure 2. The region $R=\left\{(\tau, \theta) \mid \tau_{+}<\tau<\tau_{-}\right.$and $\left.l(\tau)<\theta<m(\tau)\right\}$ is negatively invariant under the flow induced by (1.6). Also, any trajectory $Z(\xi)$ which starts in $R$ must be monotone $\left(\theta\right.$ increasing, $\tau$ decreasing) and tend to $Z_{-}$as $\xi \rightarrow-\infty$.

Consider a vertical line segment crossing $R$,

$$
\left\{(\tau, \theta) \mid \tau=\tau_{0}, l(\tau)<\theta<m(\tau)\right\}, \quad \tau_{+}<\tau_{0}<\tau_{-} .
$$

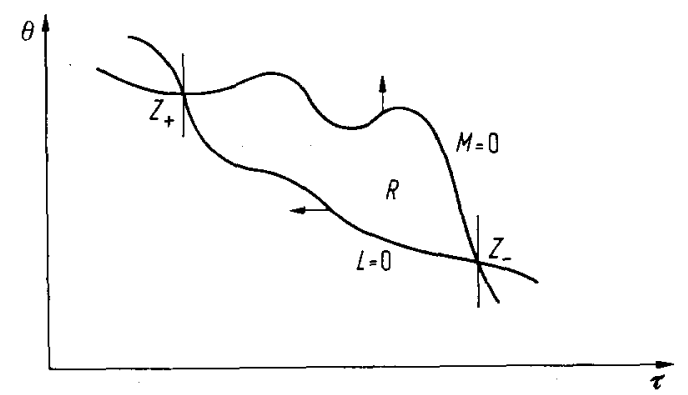

Fig. 2. Phase portrait of (1.6) for a compressive shock

A point on this segment belongs to one of three disjoint classes. In particular the forward trajectory of (1.6) starting at the point may

(a) exit the region $R$ on the curve $L=0$,

(b) exit the region $R$ on the curve $M=0$,

(c) not exit the region $R$.

In the last case, the trajectory must tend toward $Z_{+}$by monotonicity. The first two classes are open (continuity) and non-empty (endpoints). The third class is therefore also non-empty by connectedness, so some shock layer exists.

We next demonstrate the uniqueness of the shock layer. First we claim that any trajectory of (1.6) joining $Z_{+}$and $Z_{-}$must lie entirely in the region $R$. In- 
deed, no trajectory can approach $Z_{+}$within either of the regions $\{M<0$ and $L<0\}$ or $\{M>0$ and $L>0\}$. Also, the region

$$
R_{1}=\left\{(\tau, \theta) \in \Omega_{\theta} \mid \tau \leqq \tau_{+} \text {and } L>0 \text { or } M>0\right\}
$$

is negatively invariant. Thus the claim is proved.

The characteristic equation for (1.6) at the critical point $Z_{+}$always has one positive and one non-positive root

$$
\begin{aligned}
0 & =\left|\begin{array}{ll}
M_{\tau} / \mu-\varkappa & M_{\theta} / \mu \\
L_{\tau} / \lambda & L_{\theta} / \lambda-\varkappa
\end{array}\right| \\
& =\varkappa^{2}-\left(M_{\tau} / \mu+L_{\theta} / \lambda\right) \varkappa+\left[l^{\prime}\left(\tau_{+}\right)-m^{\prime}\left(\tau_{+}\right)\right] M_{\theta} L_{\theta} / \mu \lambda .
\end{aligned}
$$

The constant term is non-positive. If it is zero, then $M_{\tau}>0$. In general, then, $Z_{+}$is a saddle point, and the uniqueness of the trajectory approaching $Z_{+}$from within $R$ is easily established. In the degenerate case, one may construct a one dimensional center-stable manifold in a neighborhood of $Z_{+}$, locally invariant under (1.6). A trajectory starting in this neighborhood which does not lie in the given center-stable manifold must eventually (for $\xi$ large) leave any sufficiently small neighborhood of $Z_{+}$(see KeLLEY [3]). Therefore, any two trajectories approaching $Z_{+}$from within $R$ must lie on the same curve.

We have established the existence and uniqueness of the shock layer when $\tau_{-}>\tau_{+}$and the entropy condition $(E)_{s}$ is satisfied. If $\tau_{-}>\tau_{+}$but the condition $(E)_{s}$ fails to hold, arguments similar to those above show that no trajectory of (1.6) can connect $Z_{-}$to $Z_{+}$. Theorem 2 (and Theorem 1 for compressive shocks) is thus proved.

For a rarefaction shock joining $Z_{+}$and $Z_{-}$with $\tau_{-}<\tau_{+}, s<0$, satisfying the entropy condition $(E)_{s}$, we have $L(Z)<0<M(Z)$ for $Z$ between $Z_{+}$and $Z_{-}$on the Hugoniot curve with center $Z_{-}$. Hence $m(\tau)<l(\tau)$ for $\tau_{-}<\tau<\tau_{+}$. If $l^{\prime}(t)<0$, then the region

$$
R=\left\{(\tau, \theta) \mid \tau_{-}<\tau<\tau_{+} \text {and } m(\tau)<\theta<l(\tau)\right\}
$$

is negatively invariant and the existence and uniqueness of a trajectory connecting $Z_{-}$to $Z_{+}$is established as in the compressive case above. However, it no longer follows from (2.7) that $l^{\prime}(\tau)<0$ if $l(\tau)>m(\tau)$. On the other hand, from (2.2) follows

$$
\begin{aligned}
-\frac{1}{s} L_{\tau}(\tau, \theta) & =e_{\tau}(\tau, \theta)+p(\tau, \theta)+s M(\tau, \theta) \\
& =e_{\tau}(\tau, \theta)+p(\tau, m(\tau)) .
\end{aligned}
$$

Hence the condition $e_{\tau}(\tau, \theta) \geqq 0$ ensures that $l^{\prime}(\tau)<0$ for all $\tau$, and Theorem 1 follows.

We conclude by remarking that it is easy to use Proposition 2 and identities (2.7), (2.8) to show that a simple jump solution admits a purely viscous shock layer $(\lambda=0)$ if and only if the entropy condition $(E)_{s}$ is satisfied. This recovers the result of LiU [4], under the assumptions (1.8)-(1.10), which are different than those imposed by LIU [5]. 


\section{A shock without a shock layer}

In this section we explicitly construct an equation of state satisfying assumptions (1.8)-(1.10), and (1.14), (1.15), defined in a suitable region of the $(\tau, \theta)$-plane but violating the restriction $e_{x}(\tau, \theta) \geqq 0$, such that a (large amplitude) rarefaction shock exists satisfying the entropy condition $(E)_{s}$, while at the same time no shock layer exists if $\lambda / \mu$ is sufficiently large. The shock layer does exist if $\lambda / \mu$ is small, and entropy does increase across this shock, i.e., $S\left(Z_{-}\right)<S\left(Z_{+}\right)$with $s<0$.

Our goal is illustrated by Figure 3, representing a phase portrait for (1.6). The idea is that if $\lambda / \mu$ is large the vector field $(\tau M / \mu, \tau L / \lambda)$ for (1.6) is nearly horizontal, and a trajectory leaving $Z_{0}$ must hit the "hump" in the curve $M=0$. Then it is easy to show that there can be no trajectory connecting $Z_{-}$to $Z_{+}$. Finally using (2.6) and (2.7), one may easily check that the entropy condition $(E)_{s}$ is satisfied for the jump determined by $Z_{+}, Z_{-}$.

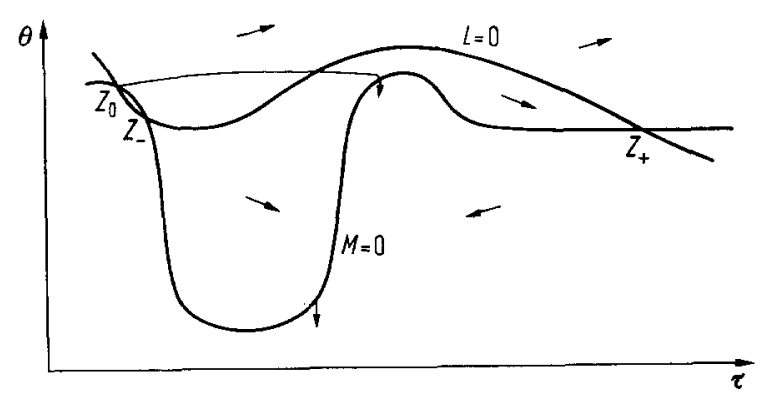

Fig. 3. A shock without a profile

Our procedure is to specify selected data (in particular the curve $\theta=m(\tau)$ on which $M=0$ ), using thermodynamic identities to make certain that the equation of state is consistent. Fix $Z_{0}=\left(\tau_{0}, \theta_{0}\right)$ in the first quadrant. We shall suppose the curve $M=0$ has the form $\theta=m(\tau)$, where $m(\tau)$ satisfies conditions $(m 1)-(m 3)$ below. (so its graph has the form shown in Figure 3 ). Later, we shall specify $p_{0}=p\left(\tau_{0}, \theta_{0}\right)$ and $s$. Then we will know $p$ along the curve $M=0$, namely

$$
p_{m}(\tau) \equiv p(\tau, m(\tau))=p_{0}-s^{2}\left(\tau-\tau_{0}\right) .
$$

For all $(\tau, \theta)$, we specify

$$
\theta p_{\theta}(\tau, \theta)=e_{\tau}(\tau, \theta)+p(\tau, \theta)=\frac{1}{\theta},
$$

so that $p_{\theta}=1 / \theta^{2}$ and (1.10) holds. The equation of state for $p$ is now determined by

$$
p(\tau, \theta)=p_{m}(\tau)+(1 / m(\tau)-1 / \theta)
$$


Our construction will be complete once $e_{\theta}(\tau, \theta)$ is determined. Indeed, since $p_{0}, s$, and $m(\tau)$ determine $p$, the $e_{\tau}(\tau, \theta)$ is then known from (3.2). Finally $e_{\tau}$ and $e_{\theta}$ determine $e(\tau, \theta)$, and $S(\tau, \theta)$ is found from the Gibbs relation (1.2). Note that (3.2) ensures that the expression obtained for $d S$ can be integrated.

We must have $\left(e_{\theta}\right)_{\tau}=\left(e_{\tau}\right)_{\theta}=-2 / \theta^{2}$, so $e_{\theta}$ has the form

$$
e_{\theta}(\tau, \theta)=e_{\theta}\left(\tau_{0}, \theta\right)-2\left(\tau-\tau_{0}\right) / \theta^{2} .
$$

We specify $e_{\theta}\left(\tau_{0}, \theta\right)$ so that (1.8) holds in the region of interest. It suffices to define

$$
e_{\theta}\left(\tau_{0}, \theta\right)=80 \tau_{0}\left(\theta^{-2}+\theta_{0}^{-2}\right),
$$

so that $e_{\theta}(\tau, \theta)>0$ for $\theta>0,0<\tau<40 \tau_{0}$. An explicit formula for $e(\tau, \theta)$ is now

$$
\begin{aligned}
e(\tau, \theta)= & 80 \tau_{0}\left(\theta_{0}^{-2}-\theta^{-2}\right) \theta-\int_{\tau_{0}}^{\tau} d \tau / m(\tau) \\
& +\left(\tau-\tau_{0}\right)\left[2 / \theta-p_{0}+\frac{1}{2} s^{2}\left(\tau-\tau_{0}\right)\right]+e_{0}
\end{aligned}
$$

We must now show that $m(\tau), p_{0}$ and $s$ may be specified so that the phase space given in Figure 3 occurs and (1.9), (1.11), (1.14) and (1.15) hold. Observe that $-s M(\tau, \theta)$ is independent of $p_{0}$ and $s$, since

$$
-s M(\tau, \theta)=p(\tau, \theta)-p_{m}(\tau)=1 / m(\tau)-1 / \theta .
$$

Using (2.7), we must have

$$
-\frac{1}{s} L_{\tau}(\tau, \theta)=\theta p_{0}-(-s) M=2 / \theta-1 / m(\tau) .
$$

Requiring $L\left(\tau_{0}, \theta_{0}\right)=0$, we see that the quantity $-L(\tau, \theta) / s$ is independent of $p_{0}$ and $s$ and recalling $-L_{\theta} / s=e_{\theta}$, namely

$$
-\frac{1}{s} L(\tau, \theta)=80 \tau_{0}\left(\theta_{0}^{-2}-\theta^{-2}\right) \theta+2\left(\tau-\tau_{0}\right)-\int_{\tau_{0}}^{\tau} d \tau / m(\tau) .
$$

Set $\tau_{k}=(k+1) \tau_{0}$. We shall show that $m(\tau)$ can be chosen so that

(i) $L(\tau, m(\tau))>0 \quad$ for $\tau-\tau_{0}>0$ small,

(ii) $L\left(\tau, \theta_{0}+\varepsilon_{1}\right)<0 \quad$ for $\tau_{3}<\tau<\tau_{5}$, for some $\varepsilon_{1}>0$,

$$
\theta_{0}<m(\tau) \leqq \theta_{0}+\varepsilon_{1} \quad \text { for } \tau_{4}<\tau<\tau_{5}
$$

(iii) $L(\tau, m(\tau))>0 \quad$ for $\tau$ large.

We ask that $m(\tau)$ be defined and smooth for $\frac{1}{2} \tau_{0}<\tau<40 \tau_{0}$, and satisfy conditions $(\mathrm{ml})-(\mathrm{m} 3)$ below. We introduce the conditions separately as follows.

$$
m\left(\tau_{0}\right)=\theta_{0} \quad \text { and } \quad m^{\prime}\left(\tau_{0}\right)=0 .
$$


Since $-L_{\tau}\left(\tau_{0}, \theta_{0}\right) / s=1 / \theta_{0}>0$, this establishes (3.6i).

$$
\begin{array}{cl}
m^{\prime}(\tau)<0 & \text { for } \tau_{0}<\tau<\tau_{1}, \\
m(\tau)=\theta_{0} / 6 & \text { for } \tau_{1}<\tau<\tau_{3}, \\
m^{\prime}(\tau)>0 & \text { for } \tau_{3}<\tau<\tau_{4},
\end{array}
$$

$\max _{\tau_{4} \leqq \tau \leqq \tau_{5}} m(\tau)=\theta_{0}+\varepsilon_{1}, \quad$ for some $\varepsilon_{1}>0$ small, with $m\left(\tau_{4}\right)=\theta_{0}=m\left(\tau_{5}\right)$.

To verify (3.6ii), we have for $\tau_{3}<\tau<\tau_{5}$ and $\varepsilon_{1}$ small

$$
\begin{gathered}
-\frac{1}{s} L\left(\tau, \theta_{0}+\varepsilon_{1}\right) \leqq C \varepsilon_{1}+2\left(\tau_{5}-\tau_{0}\right) / \theta_{0}-\left(\tau_{3}-\tau_{1}\right) 6 / \theta_{0} \\
=C \varepsilon_{1}-2 \tau_{0} / \theta_{0}<0 \\
m(\tau)=\theta_{0} \quad \text { for } \tau>\tau_{5} .
\end{gathered}
$$

To verify (3.6iii), we have for $\tau>\tau_{6}$

$$
\begin{aligned}
-\frac{1}{s} L\left(\tau, \theta_{0}\right) & \geqq 2\left(\tau-\tau_{0}\right) / \theta_{0}-\left[\left(\tau_{4}-\tau_{0}\right) 6 / \theta_{0}+\left(\tau-\tau_{4}\right) / \theta_{0}\right] \\
& =\left[\left(\tau-\tau_{4}\right)-4\left(\tau_{4}-\tau_{0}\right)\right] / \theta_{0}>0
\end{aligned}
$$

if $\tau>\tau_{20}$.

Since $-L_{\theta}(\tau, \theta) / s \geqq c>0$ for $\frac{1}{2} \tau_{0}<\tau<\tau_{40}$, the set $\{L=0\}$ is a curve $\theta=l(\tau)$ which is bounded above. We claim that $l(\tau)$ is defined for $\tau_{0} \leqq \tau \leqq \tau_{5}$. Indeed, $L\left(\tau, \theta_{0}\right)<0$ for $\tau_{3}<\tau<\tau_{5}$, and since $L_{\tau}\left(\tau, \theta_{0} / 6\right)>0$ for $\tau<\tau_{3}$ (use (2.7) with $M \leqq 0$ ) we have $L\left(\tau, \theta_{0} / 6\right)<0$ for $\tau<\tau_{3}$. Hence

$$
l(\tau)>m(\tau)
$$

for $\tau_{1}<\tau<\tau_{5}$, so that $l(\tau)$ is bounded below.

We now set

$$
\begin{aligned}
& \tau_{+}=\min \left\{\tau \mid \tau \geqq \tau_{5} \text { and } l(\tau)=m(\tau)\right\} \\
& \tau_{-}=\max \left\{\tau \mid \tau \leqq \tau_{1} \text { and } l(\tau)=m(\tau)\right\}
\end{aligned}
$$

Then $\tau_{5}<\tau_{+}<\tau_{20}$, and since $m^{\prime}\left(\tau_{0}\right)=0>l^{\prime}\left(\tau_{0}\right)$ we have also $\tau_{0}<\tau_{-}<\tau_{1}$. Finally we set $\theta_{+}=m\left(\tau_{+}\right), \theta_{-}=m\left(\tau_{-}\right)$, fixing $Z_{+}$and $Z_{-}$and completing the construction of Figure 3 .

It remains to choose $p_{0}$ and $s$ so that $p(\tau, \theta)>0$ and (1.9), (1.14), (1.15) hold in a suitable domain. But

$$
p_{\tau}(\tau, S)=p_{\tau}(\tau, \theta)-\theta p_{\theta}(\tau, \theta)^{2} / e_{\theta}(\tau, \theta)
$$

as follows by calculations $\partial p(\tau, S(\tau, \theta)) / \partial \tau$ and using the identities (2.1), (2.2) and the relation $\theta_{\tau}(\tau, S)=-S_{\tau}(\tau, \theta) / S_{\theta}(\tau, \theta)$. Thus (1.15) implies (1.9). But from (3.2) we have

$$
p_{z}(\tau, \theta)=-s^{2}+(1 / m(\tau))^{\prime}
$$

so that $p_{\tau}(\tau, \theta) \leqq 0$ for $0<\tau<\tau_{40}$ if we choose $s^{2}$ sufficiently large. 
Along with the choice of $p_{0}$, we shall fix the domain of states $\Omega_{p}$ in the $(\tau, p)$ plane so that $\Omega_{p}$ is convex, $p>0$ in $\Omega_{p}$, the condition (1.14) holds, and the image domain $\Omega_{\theta}$ in the $(\tau, \theta)$-plane contains (say) the half strip $\left\{(\tau, \theta) \mid 0<\tau<\tau_{40}\right.$, $\left.\theta>\theta_{0} / 10\right\}$. It suffices to choose

with

$$
\Omega_{p}=\left\{(\tau, p) \mid 0<\tau<\tau_{40} \text { and } p-p_{0}+s^{2}\left(\tau-\tau_{0}\right)>-10 / \theta_{0}\right\}
$$

$$
p_{0}=40 \tau_{0} s^{2}+10 / \theta_{0} .
$$

To check (1.14), we note that

$$
e_{\tau}(\tau, p)=e_{\tau}(\tau, \theta)+e_{\theta}(\tau, \theta)\left[-p_{\tau}(\tau, \theta) / p_{0}(\tau, \theta)\right] .
$$

Then using (3.2) and (3.8), it follows that $e_{\tau}(\tau, p) \geqq 0$ in $\Omega_{p}$ if $s^{2}$ is sufficiently large.

Now the equation of state is completely specified, and except for (1.12) all the conditions (1.8)-(1.15) hold. We have already noted that the entropy condition $(E)_{s}$ holds, since $l(\tau)>m(\tau)$ for $\tau_{-}<\tau<\tau_{+}$

The penultimate stage of our analysis is to show that, for $\lambda / \mu$ sufficiently large, there is a trajectory of (1.6) leaving $Z_{0}$ and intersecting the curve $M=0$ before the "hump". Since $m^{\prime}\left(\tau_{0}\right)>l^{\prime}\left(\tau_{0}\right)$, it is clear from (2.9) that $Z_{0}$ is a saddle point for (1.6). If $\varkappa$ is the positive characteristic value for (1.6) at $Z_{0}$, the characteristic vector $\left(\varkappa-\tau L_{\theta} / \lambda, \tau L_{\tau} / \lambda\right)$ may be shown to have positive components. Let $Z(\xi)$ be the solution leaving $Z_{0}$ along this vector. So long as $Z(\xi)$ remains above the curve $M=0$, the specific volume $\tau$ is increasing on $Z(\xi)$ and we may write $\theta=z(\tau)$ along $Z(\xi)$. Then

$$
\frac{d z}{d \tau}=\frac{\mu L(\tau, z(\tau))}{\lambda M(\tau, z(\tau))}
$$

It is clear that $z(\tau)$ is defined and increasing for $\tau_{0}<\tau<\tau_{*}$, where

$$
\tau_{*}=\min \left\{\tau \mid \tau>\tau_{0} \text { and } L\left(\tau, \theta_{0}\right)=0\right\} \text {. }
$$

Note that $\tau_{*}<\tau_{3}$.

We shall "channel" the curve $z(\tau)$ under the curve $L=0$ by using a thin box with a corner cut out. To this end, define

$$
R^{*}=\left(\tau_{0}, \tau_{3}\right) \times\left(\theta_{0}-\varepsilon_{2}, \theta_{0}+\varepsilon_{1}\right) \backslash\left(\tau_{0}, \tau_{*}\right) \times\left(\theta_{0}-\varepsilon_{2}, \theta_{0}+\frac{1}{2} \varepsilon_{1}\right) .
$$

Here the constant $\varepsilon_{2}>0$ is chosen so that $\bar{R}^{*}$ is contained in the region $\{M>0\}$. Now, the trajectory $Z(\xi)$ must enter $R^{*}$ on the cut-out part of the boundary with $\theta>\theta_{0}$, i.e., it must enter at a point $\left(\tau, \theta_{0}+\frac{1}{2} \varepsilon_{1}\right)$ for $\tau_{0}<\tau \leqq \tau_{*}$, or at a point $\left(\tau_{*}, \theta\right)$ for $\theta_{0}<\theta \leqq \theta_{0}+\frac{1}{2} \varepsilon_{1}$. then

Since $\sup |L / M|$ is finite, it follows that if $\lambda / \mu$ is sufficiently large in $R^{*}$

$$
\left|\frac{d z}{d \tau}\right| \leqq \min \left\{\varepsilon_{1} / 2\left(\tau_{3}-\tau_{0}\right), \varepsilon_{2} /\left(\tau_{3}-\tau_{*}\right)\right\} \quad \text { for } Z(\xi) \in R^{*} .
$$

This implies that the trajectory $Z(\xi)$ must leave $R^{*}$ on its right boundary, $\left\{\tau_{3}\right\} \times\left(\theta_{0}-\varepsilon_{2}, \theta_{0}+\varepsilon_{1}\right)$, which is contained in the region $\{L<0$ and $M>0\}$. 
Afterwards $\theta$ decreases and $\tau$ increases along $Z(\xi)$. Therefore, since $m(\tau)$ reaches the value $\theta_{0}+\varepsilon_{1}$, the path $Z(\xi)$ must intersect the curve $M=0$ at a point $(\tilde{\tau}, \tilde{\theta})$ where $\tau_{5}>\tilde{\tau}>\tau_{3}>\tau_{-}$.

We may now easily construct a positively invariant region $\mathscr{R}$ containing $Z_{-}$ and disjoint from $Z_{+}$, thus proving that no trajectory can connect $Z_{-}$to $Z_{+}$. The region $\mathscr{R}$ is bounded on the right by the line $\tau=\tilde{\tau}$ for $\theta \leqq \tilde{\theta}(M<0$ here), from above by the curve $\theta=z(\tau)$ for $\tau_{0}<\tau<\tilde{\tau}$ (trajectories cannot cross), and by the curve $\theta=\min \{l(\tau), m(\tau)\}$ for $\tau \leqq \tau_{0}$ (the vector field either points down, or to the left at a point where $l^{\prime}(\tau)<0$, as in section 2). This completes the construction of our example.

Acknowledgements. This work comprises one chapter of the author's Ph.D. dissertation, written with the guidance and encouragement of Professor ANDREw MAJDA at the University of California, Berkeley. The research was sponsored by the United States Army under Contract No. DAAG29-80-C-0041 and is based upon work supported by the National Science Foundation under Grant No. MCS-7927062.

\section{References}

1. C. Dafermos, Global smooth solutions for the initial boundary value problem for the equations of one dimensional nonlinear thermoviscoelasticity, SIAM J. Math. Anal. 13 (1982) 397-408.

2. D. GiLbaRG, The existence and limit behavior of the one dimensional shock layer, Amer. J. Math. 7 (1951) 256-274.

3. A. Kelley, Stability of the center-stable manifold, J. Math. Anal. Appl. 18 (1967) 336-344.

4. T.-P. LIU, The entropy condition and the admissibility of shocks, J. Math. Anal. Appl. 53 (1976) 78-88.

5. T.-P. Liv, Shock waves in the nonisentropic gas flow, J. Diff. Equs. 22 (1976) $442-452$.

6. A. Matsumura and T. Nishida, The initial value problem for the equations of motion of compressible viscous and heat-conductive fluids, Proc. Japan Acad 55, Ser. A (1979) 337-342.

7. R. PEGO, Stable viscosities and shock profiles for systems of conservation laws, Transactions AMS 282 (1984) 149-163.

8. J. SerRin, Phase transitions and interfacial layers for van der Waals fluids, in Recent Methods in Nonlinear Analysis and Applications, ed. A. Confora, et al., Instituto Mathematico, Università di Napoli, Naples (1980) 169.

9. M. SLEMROD, Admissibility criteria for propagating phase boundaries in a van der Waals fluid, to appear in Arch. Rat. Mech. Anal.

10. R. G. SмiтH, The Riemann problem in gas dynamics, Transactions of the AMS 249 (1979) $1-50$.

11. H. WeYL, Shock waves in arbitrary fluids, Comm. Pure Appl. Math. 2 (1949) 103122.

Department of Mathematics University of Michigan

Ann Arbor 